\title{
Una breve historia de las organizaciones del Movimiento Indígena del Ecuador
}

\author{
Philipp Altmann ${ }^{1}$
}

Resumen

EL MOVIMIENTO INDÍGENA DEL ECUADOR ESTÁ COMPUESTO POR DIFERENTES ORGANIZACIONES INDÍGENAS QUE COMPITEN ENTRE SÍ POR MIEMBROS Y RECURSOS. ESTOS ACTORES ENCARNAN DIFERENTES INTERESES Y TENDENCIAS QUE A VECES SON CONTRADICTORIAS, DADAS LAS DISTINTAS CONCEPCIONES POLÍTICAS, ÉTNICAS Y SOCIALES QUE SE MANIFIESTAN EN EL INTERIOR DE LAS ORGANIZACIONES, POR LO TANTO HABLAR DEL MOVIMIENTO INDíGeNa SIN CONSIDERAR ESTA DIVERSIDAD ORGANIZATIVA RESULTARÍA UN ANÁLISIS LIMITADO.

ESTE ARTíCULO INTENTARÁ HACER UN RESUMEN HISTÓRICO DE LAS ORGANIZACIONES INDÍGENAS, DESTACANDO SUS DEMANDAS, SUS ESTRATEGIAS PARTICULARES Y LAS RELACIONES QUE EXISTEN ENTRE ELLAS. NO VAMOS A INTERPRETAR EL DESARROLLO DEL MOVIMIENTO COMO SUCESIÓN DE ORGANIZACIONES CON IDEOLOGÍAS GENERALMENTE INCOMPATIBLES, SINO COMO UNA DINÁMICA DE COMPETENCIA Y A VECES COOPERACIÓN ENTRE LAS DISTINTAS ORGANIZACIONES.

ESTE ESTUdio SE BASA EN PUBliCACIONES REALIZAdAS POR LAS PROPIAS ORGANIZACIONES, COMPLETÁNDOLAS Y AMPLIÁNDOLAS CON TEXTOS ACADÉMICOS, CON LA INTENCIÓN DE AYUDAR A LA SISTEMATIZACIÓN DE UNA AUTO-HISTORIA DEL MOVIMIENTO INDÍGENA DESDE UNA PERSPECTIVA CIENTÍFICA.

Palabras Clave: movimiento indígena del ecuador - Organizaciones indígenas ecuatorianas.

Abstract

THE INDIGENOUS MOVEMENT OF ECUADOR IS COMPOSED OF DIFFERENT INDIGENOUS ORGANIZATIONS THAT COMPETE WITH EACH OTHER FOR MEMBERS AND RESOURCES. THESE ACTORS EMBODY DIFFERENT INTERESTS AND TENDENCIES THAT ARE OCCASIONALLY CONTRADICTORY, GIVEN THE DIVERSITY OF POLITICAL, ETHNIC, AND SOCIAL CONCEPTIONS EXPRESSED WITHIN THE ORGANIZATIONS. TO SPEAK OF THE INDIGENOUS MOVEMENT WITHOUT CONSIDERING THIS DIVERSITY OF PERSPECTIVES WOULD RESULT IN A LIMITED ANALYSIS.

This aRTICle ATTEMPTS TO CREATE A HISTORICAL REVIEW OF THESE INDIGENOUS ORGANIZATIONS HighLIGHTING THEIR DEMANDS, THEIR PARTICULAR STRATEGIES AND THEIR RELATIONSHIPS TO ONE ANOTHER. THE DEVELOPMENT OF INDIGENOUS MOVEMENT IS NOT UNDERSTOOD AS A SUCCESSION OF ORGANIZATIONS WITH INCOMPATIBLE IDEOLOGIES BUT INSTEAD AS A DYNAMIC OF COMPETITION AND SOMETIMES COOPERATION BETWEEN THE ORGANIZATIONS.

THIS STUDY IS BASED ON PUBLICATIONS CREATED BY THE ORGANIZATIONS THEMSELVES, COMPLEMENTED BY ACADEMIC TEXTS WITH THE GOAL OF AIDING THE SYSTEMATIZATION OF AN “AUTO"-HISTORY OF THE INDIGENOUS MOVEMENT FROM A SCIENTIFIC PERSPECTIVE.

KEY WORDS: INDIGENOUS MOVEMENT OF ECUADOR - ECUADORIAN INDIGENOUS ORGANIZATIONS.

1 PhilippAltmann@gmx.de 


\section{Introducción}

$\mathrm{L}$ as organizaciones del movimiento indígena del Ecuador tienen una estructura "piramidal" (Bretón 2003: 219) cuya base son las organizaciones locales, de primer grado (OPG) o de base (comunidades, cooperativas, asociaciones, centros u otras organizaciones de pequeños grupos). Las organizaciones de primer grado están agrupadas a nivel provincial en organizaciones de segundo grado (OSG). Éstos, por su parte, están agrupadas en federaciones a nivel regional o de tercer grado (OTG), las plataformas regionales de la Confederación de Nacionalidades Indígenas del Ecuador (CONAIE) -ECUARUARI (Ecuador Runacunapak Rikcharimui, El Despertar de los Indígenas Ecuatorianos), CONFENIAE (Confederación de Nacionalidades Indígenas de la Amazonía Ecuatoriana) y CONAICE (Confederación de Nacionalidades Indígenas de la Costa Ecuatoriana)- o directamente las federaciones nacionales en el caso de la Federación Ecuatoriana de Indios (FEI), la Confederación de Organizaciones Campesinas, Indígenas y Negras (FENOCIN) o el Consejo Ecuatoriano de Pueblos y Organizaciones Indígenas Evangélicos (FEINE) (Bretón, en Wolff, 2008: 120).

El sistema piramidal no es jerárquico, las organizaciones de primer y segundo grado tienen una autonomía considerable hasta el punto que militan al mismo tiempo en diferentes organizaciones regionales y nacionales, o cambian de una organización a la otra, siguiendo sus intereses particulares (Botero, 1998: 9). Además, no existe afiliación individual en ninguna de las organizaciones indígenas. La adhesión se realiza por la participación activa en las OPG o la designación por ellas en una OSG u OTG (León, 1991: 402). Aquí, se limita al análisis de las organizaciones regionales y nacionales del movimiento indígena, dejando al lado las organizaciones de niveles inferiores.

Durante el desarrollo del movimiento indígena del Ecuador, se puede notar una pérdida de representatividad de las organizaciones sindicales con enfoque de clase, como la FEI y la FENOCIN. A partir de los años 1970 surge con más fuerza la tendencia étnica, en la Federación Shuar, ECUARUNARI y después CONAIE, que pone énfasis en la lucha por los intereses étnicos de los indígenas, sin perder por completo la idea de clase (Bretón, 2003: 228). No obstante, existen críticas a esta manera de entender el desarrollo del movimiento indígena. Marc Becker (2007) sostiene que la FEI ya tenía una concepción del racismo y de la importancia de lo étnico. A continuación, se detalla el estudio de las organizaciones particulares categorizado de una forma histórica según fechas de fundación.

\section{La Federación Ecuatoriana de Indios (FEI)}

\section{Fundación}

Los años 1920 y 1930 han sido caracterizados por una progresiva politización de la población campesina. Esto se debe en parte a un auge de las tendencias socialistas e intentos de organización de los campesinos por los partidos socialista (fundado en 1925) y comunista (fundado en 1931) (Büschges, 2009: 45). A partir de 1926, influyeron en la fundación de los sindicatos: El Inca de Pesillo, Tierra Libre de Moyurco, Pan y Tierra de la Chimba, y un sindicato en Juan Montalvo, todos en el sector de Cayambe, Provincia de Pichincha (Becker, 1997: 169-170). Un primer intento de realizar un congreso indígena en Juan Montalvo en 1931 es impedido por el ejército (Tamayo, 1996: 89). En 1934 se pudo realizar la Conferencia de Cabecillas Indígenas en la Casa del Obrero en Quito, siendo el primer paso para la formación de una organización indígena nacional. Una reacción del Estado hacia esta creciente movilización fue la Ley de Comunas de 1937, que buscó establecer un nuevo sistema de control sobre los indígenas (CONAIE, 1989: 31) - y terminó construyendo la base para la futura expansión del movimiento indígena. 
La publicación más importante de los indígenas en esos años era el boletín Nucanchic Allpa ("Nuestra tierra"), autonombrado "Órgano de los sindicatos, comunidades e indios, en general" que se publicó desde 1930 y se convirtió en 1944 en el portavoz de la FEI, función que cumplió hasta el fin de su publicación en 1968 (Albó, 2009: 205-206).

En agosto de 1944, después de la revolución que derrocó al gobierno de Arroyo del Río (Tamayo, 1996: 89), se fundó el sindicato marxista Confederación de Trabajadores del Ecuador (CTE), cercano al Partido Comunista del Ecuador (PCE), y su filial campesina, la Federación Ecuatoriana de Indios (FEI) (Kaltmeier, 2009: 344). El 29 Enero de 1945, la FEI es reconocida mediante acuerdo ministerial (FEI s.f.). Ambas organizaciones sindicales lucharon contra el sistema hacendado y por una reforma agraria. La FEI sigue siendo parte de la CTE hasta hoy (Büschges, 2009: 54), lo que le pone en un contexto de izquierda comunista también internacionalmente, mediante la membresía de la CTE en la Federación Sindical Mundial, de tendencia comunista.

La cercanía de la FEI al PCE ha sido interpretada muchas veces como una falta de autonomía de la organización indígena. La influencia del PCE que sin duda hubo (Tamayo, 1996: 89) es entendida como "control" (GTZ, 2004: 3) o como una imposición desde afuera (Moreno/ Figueroa, 1992: 33-34). Santana describe la FEI como "la rama campesina del movimiento de masas del PCE" (Santana, 1983: 159), creada con este fin explícito. La FEI sigue una "estrategia de de-etnizar la movilización política [y considera; el autor] la población indígena como parte del campesinado nacional, sin considerar cuestiones étnicas" (Büschges, 2009: 45). Sin embargo, intenta "vincular la lucha de clases con la defensa de derechos indígenas particulares" (Büschges, 2009: 45), lo que permite considerarla una organización propiamente indígena, y no sólo una marioneta del PCE.

La influencia del PCE en la FEI se deja explicar mejor por la inclusión institucional en la CTE y por el -en parte- mismo personal de las dos organizaciones. Esta línea de explicación propone Becker (2007: 139), quien destaca el rol del PCE en la formación de líderes indígenas. También otras organizaciones del movimiento indígena admiten que la politización y formación de los indígenas y de los futuros líderes haya sido una contribución importante de la FEI al desarrollo del movimiento indígena (ECUARUNARI/CONAIE, 1989: 40). Así, "el Partido Comunista no formó el movimiento indígena, sino que los dos nacieron de la misma lucha" (Becker, 2007: 139). La relación estrecha de la FEI en con la CTE permitió una lucha tanto en el campo, mediante las conexiones locales de los dirigentes indígenas, como en las ciudades, con apoyo de los comunistas urbanos (Becker, 2007: 140).

La fuerza organizativa de la FEI estaba presente especialmente en la Sierra, sobre todo en las provincias de Cayambe, Cotopaxi y Chimborazo (Kaltmeier, 2009: 344), hecho en estrecha relación con su orientación hacia los trabajadores agrarios (Tamayo, 1996: 64).

\section{Principios y Estrategia}

La FEI estaba ligada a la lucha de los huasipungueros y trabajadores de haciendas, por esto sus demandas se basaron en la mejora de las condiciones de trabajo y de los salarios (Becker, 2007: 138-139). Desde el principio exigió una redistribución de la tierra, y finalmente "tuvo notable participación en las dos reformas agrarias [de 1964 y 1973; el autor]" (Guamán, 2003: 31) La lucha por los derechos de los indígenas y contra la discriminación (Minkner-Brünjer, 2009: 138). era otra temática importante. Pero, según la CONAIE “[1]a FEI no tomó en cuenta la globalidad de nuestros problemas, este es, la explotación de clase y la discriminación étnica a la que estamos sujetos" (CONAIE, 1989: 31-32). Para la FEI "la cuestión étnica [...] no constituye un problema político" (Santana, 1983: 162), punto de vista que difiere del de muchos indígenas.

Estudios recientes muestran a la FEI más abierta a una perspectiva cultural. Un índice de esta apertura es el trabajo educativo de la FEI, sobre todo el de su directora, Tránsito Amaguaña, quien inició escuelas campesinas clandestinas donde se enseñó en kichwa (Becker, 1997: 296). De una 
organización de clase con enfoque étnico hablan también los principios de 1945 de la FEI, que proponen "la emancipación económica de los indios ecuatorianos" (en: Becker, 2007: 141). Por esta razón, Becker llega a la conclusión que "Ideologías de clase, etnicidad y nacionalidad estaban todas presentes en varias formas y en varios niveles en la fundación de la FEI, con activistas dando énfasis a distintos aspectos para encontrarse con las necesidades y demandas actuales" (Ibíd., 2007: 141). Esta orientación de clase con enfoque étnico sigue vigente, como demuestra la autopresentación de la FEI en las páginas web de la CTE. Las demandas de igualdad económica y social se ven completadas por los fines constituidos de la FEI que al lado de "la igualdad social, política, económica" (FEI s.f.) pone énfasis a "las diferencias de identidad cultura y lengua" (FEI s.f.). Esto implica una defensa y valoración de las nacionalidades y pueblos indígenas, como de sus organizaciones (FEI s.f.).

\section{Desarrollo}

Aunque nunca pudo reunir a la mayoría de la población indígena, la FEI, como la primera asociación indígena a nivel nacional, ha tenido una considerable fuerza organizativa y contribuyó a la politización y concientización en los indígenas de la Sierra. Una prueba de esto es su VI. Congreso en 1952 con cuatrocientos delegados de seis provincias de la Sierra (Albornoz, 1976: 151). Los años 1950 fueron la época más activa de la FEI, hecho que está en estrecha relación con el desarrollo del capitalismo en la Sierra ecuatoriana (CONAIE, 1989: 32). En esa época, la FEI pudo integrarse organizativamente en las comunidades (Botero, 1998: 8).

En los años 1960 empezó la crisis de la FEI. En 1961, la FEI fue capaz de organizar una manifestación de cerca de 12,000 huasipungueros en el centro de Quito (Becker, 1997: 298), la mayor manifestación campesina hasta ése momento. Pero en ése mismo año "[e]1 Secretario General del PCE se quejaba [...] que la FEI había dejado de funcionar y que era necesario reemprender el trabajo" (Santana, 1983: 159). La ejecución de las demandas de la FEI, como las reformas agrarias de 1964 y 1973, la dejaron sin base para operar (CONAIE, 1989: 33). Hasta los años 1970, la FEI fue gradualmente reemplazada por la FENOC (Federación Nacional de Organizaciones Campesinas) como actor importante en la Sierra ecuatoriana (Kaltmeier, 2009: 344). Ya a fines de la década de los 1970, la FEI se mantuvo "difícilmente entre sectores minoritarios del campesinado beneficiario de la reforma agraria" (Santana, 1983: 159). Sólo pudo realizar acciones grandes en cooperación con otras organizaciones por ejemplo en el Frente Único de Lucha Campesina e Indígena (FULCI) a fines de los 1970 (Rodríguez, 2008: 91) y la participación en acciones de la CONAIE en los tardíos 1980 y tempranos 1990.

La FEI ha sufrido pero sigue con vida como demuestran sus acciones que -en conjunto con la CTE u organizaciones indígenas más grandes- sigue realizando. No obstante, quizá su autodescripción más actual sea exagerada: "Para el año 2006 la FEI es una organización con presencia orgánica en todas las provincias del Ecuador; generando políticas que infieren con el quehacer nacional" (FEI, s.f.). Según datos del Consejo de Desarrollo de las nacionalidades y pueblos del Ecuador (CODENPE) de 2007, la FEI tiene dos OSG en las provincias de Chimborazo y Esmeraldas y un total de 5.655 miembros (IEE, s.f.).

\section{La Confederación de Organizaciones Campesinas, Indígenas y Negras (FENOCIN)}

\section{Fundación}

En 1938 se constituyó la Confederación Ecuatoriana de Obreros Católicos (CEDOC) como un intento de la Iglesia Católica y del Partido Conservador de crear un sindicato ajeno a las tendencias de la izquierda (CONAIE, 1989: 32). Agrupaba sobre todo a artesanos y obreros urbanos (Albó, 2009: 207). En 1974 cambia su nombre a Confederación de Organizaciones Clasistas y a partir de 1988 se reconstituye como Confederación de Organizaciones Clasistas 
Unitarias de Trabajadores (CEDOCUT) a la cual sigue perteneciendo la FENOCIN (Tamayo, 1996: 89-90).

La CEDOC refuerza su presencia en el campo a partir de los años 1950 (Santana, 1983: 159). Durante la reforma agraria crea su filial Federación de Trabajadores Agropecuarios (FETAP) “el 9 de marzo de 1965, con la concurrencia de delegados de 26 organizaciones de base" (FENOCIN, 2004: 7). La FETAP lucha contra el huasipungaje, por derechos laborales y una redistribución de la tierra, hecho que contribuyó a su rápido crecimiento. En su tercer Congreso, en noviembre de 1968, con 200 delegados de las organizaciones de base, la FETAP decidió cambiar su nombre a Federación Nacional de Organizaciones Campesinas (FENOC) (FENOCIN, 2004: 8) y de encabezar la lucha por una reforma agraria. En este contexto, la FENOC desarrolla una gran capacidad de movilización (Chiriboga, 1986: 68).

\section{Desarrollo}

A principios de los años 1970 una nueva tendencia reformista y demócrata cristiana empezó a reemplazar a la tendencia conservadora original en la FENOC y la CEDOC. Por estas divergencias, algunas organizaciones se separaron de la FENOC y se unieron a la ECUARUNARI, que era más abierta hacia la teología de la liberación y tendencias afines (FENOCIN, 2004: 10). En el tercer Congreso de la FENOC, en 1973, se definió un programa de lucha por la tierra, por mejores salarios para los trabajadores rurales y por la intervención de las organizaciones campesinas en el proceso de reforma agraria (FENOCIN, 2004: 10). El último punto se reforzó con la constitución del Frente Unitario por la Reforma Agraria (FURA), en conjunto con la FEI, ECUARUNARI y otros (Chiriboga, 1986: 86; FENOCIN, 2004: 10-11). Esta clarificación de la posición de la FENOC la llevó a un auge y la consolidación de su estructura en uniones y federaciones regionales (FENOCIN, 2004: 12). En octubre de 1973 se promulga una nueva ley de reforma agraria.

A partir de 1975 los conflictos dentro de la FENOC se resuelven cuando -en la fase "caliente" de la lucha agraria- una tendencia socialista se impone (Ospina, 2008: 71). En consecuencia, la FENOC se acerca al Partido Socialista Ecuatoriano (PSE) (Barrera, s.f.: 151), alianza que permanece hasta hoy. Este mismo año, el gobierno militar detiene la reforma agraria, lo cual forzó la FENOC a cambiar su estrategia hacia una lucha sindical en conjunto con los sindicatos urbanos (Minkner-Brünjer, 2009: 139). El FURA perdió fuerza (Chiriboga, 1986: 86).

En 1977, el quinto Congreso de la FENOC pudo reunir más de 400 delegados. Se hizo evidente el peso de los minifundistas y jornaleros agrícolas de la Costa y la Sierra (FENOCIN, 2004: 14-15). Al año siguiente, las organizaciones campesinas intentan otro esfuerzo en la lucha agraria. "Se constituye inicialmente el FULC (Frente Único de Lucha Campesina) entre la FENOC, la FEI y el ECUARUNARI y posteriormente el FULCI (Frente Único de Lucha Campesina e Indígena) buscando incorporar en la acción a las organizaciones indígenas del Oriente" (FENOCIN, 2004: 17). Las demandas eran principalmente la aplicación de la reforma agraria y la reforma de las leyes de fomento agropecuario y de seguridad nacional (Rodríguez, 2008: 91). Las dos plataformas no tienen una larga vida efectiva (Chiriboga, 1986: 86).

Con el retorno a la democracia en 1979, la FENOC persigue nuevamente la reforma agraria (FENOCIN, 2004: 15). Como el gobierno Roldós/Hurtado (1979-1983) no actúa de la forma esperada, la FENOC participa en la Marcha Nacional Campesina e Indígena en octubre de 1980 y en las cinco huelgas nacionales entre mayo 1981 y marzo 1983 (FENOCIN, 2004: 15). En 1982, la FENOC participa en la creación de la Coordinadora Nacional Campesina e Indígena que agrupa a todas las organizaciones indígenas y campesinas del país y exige una "reforma agraria con control campesino" (FENOCIN, 2004: 17). En esta época, la FENOC entra en la política partidaria. En conjunto con los grandes sindicatos, el PCE y otras fuerzas, apoya la creación del Frente Amplio de Izquierda (FADI). En varias provincias postula candidatos propios (FENOCIN, 2004: 15). En 1995, el FADI se une al PSE, ahora Partido Socialista-Frente Amplio (PS-FA). 
A principios de los 1980, la FENOC es la organización campesina más grande del país. Pero los intereses diversos que representa la dirigen hacia demandas particularistas, impiden su desarrollo y la conducen a una fuerte crisis (Santana, 1983: 159). Estos problemas se agravan bajo el gobierno autoritario de Febres Cordero (1984-1988). En 1986, el sexto Congreso de la FENOC reúne a unos 3,000 campesinos, pero no trae innovaciones programáticas (FENOCIN, 2004: 18). Más bien marca el principio de la crisis que dura hasta mediados de los 1990. El estancamiento de la organización se agudiza. La mayor innovación de esta época es el cambio de nombre a Federación Nacional de Organizaciones Campesinas-Indígenas (FENOC-I) en 1988 (FENOCIN, 2004: 19), un reconocimiento oficial de la presencia de las organizaciones indígenas que formaron un 40\% de la FENOC en ese momento (Tamayo, 1996: 89-90).

La FENOC-I vuelve a aparecer en la esfera pública con su participación en el levantamiento de 1994. Después, con la CONAIE, FEI y FENACLE (Federación Nacional de Campesinos Libres del Ecuador) conformó la Coordinadora Agraria Nacional (CAN) para luchar contra la Ley de Desarrollo Agrario del gobierno Durán Ballén que pretendía terminar definitivamente los procesos de reforma agraria e imponer una liberalización en el agro (FENOCIN, 2004: 19). En 1995 se realizó el séptimo Congreso de la FENOC-I, donde se enmarca una modernización profunda de la organización. "La federación se auto reconoce como pluriétnica, intercultural, democrática, que lucha por un desarrollo sustentable, equitativo, con enfoque de género y generacional" (FENOCIN, 2004: 20). Esta es la primera vez que la interculturalidad aparece en la agenda del movimiento indígena.

En 1994 participó en la fundación de la Coordinadora Latinoamericana de Organizaciones del Campo (CLOC) que en 1996 se integró a la organización campesina mundial Vía Campesina (FENOCIN, 2004: 25). En un congreso extraordinario en 1997, la FENOC-I aumenta su nombre a Federación de Organizaciones Campesinas, Indígenas y Negras (FENOCIN) y se define como "la única organización nacional que reconoce al mismo tiempo la diferencia de las identidades y la interculturalidad" (FENOCIN, 2004: 20).

Los años 1990 fueron marcadas por una creciente hegemonía de la CONAIE que se impuso poco a poco en las agencias gubernamentales de desarrollo campesino y educación intercultural, culminando en la creación del Consejo de Desarrollo de las Nacionalidades y Pueblos del Ecuador (CODENPE) en 1999 bajo el control de la CONAIE. Esto colocó a la FENOCIN en oposición a la CONAIE que se define como única representación de los indígenas (FENOCIN, 2004: 21).

En su octavo Congreso en 1999, la FENOCIN cambia su nombre a Confederación Nacional de Organizaciones Campesinas, Indígenas y Negras (FENOCIN) (FENOCIN, 2004: 33). Complementó su programa con demandas de un "desarrollo sustentable, equitativo y con identidad [y] la globalización con identidad y oposición al neoliberalismo" (FENOCIN, 2004: 25).

La FENOCIN tiene presencia en 18 de las 24 provincias de Ecuador, en la Sierra, la Costa y el Oriente. Agrupa a 61 uniones regionales y provinciales u OSG's (FENOCIN, 2004: 26), y más de 1.200 organizaciones de base (FENOCIN, 2004: 32). Según datos del CODENPE de 2007, la FENOCIN tiene 17 OSG en siete provincias (Cañar, Carchi, Esmeraldas, Imbabura, Loja, Pichincha y Azuay) y 109.278 miembros (IEE, s.f.). "Su filial más importante es la Unión de Organizaciones Indígenas y Campesinas de Cotacachi -UNORCAC- que aglutina a 43 comunidades de Cotacachi" (GTZ, 2004: 36). El peso más grande tienen las OSGs en la Sierra y -en menor medida- en la Costa, mientras que en la Amazonía, la FENOCIN sólo está presente en pocas localidades (Minkner-Brünjer, 2009: 145-146).

Entre las organizaciones del movimiento indígena, la FENOCIN es la más cercana al gobierno de Correa. El PS-FA está involucrado en el actual gobierno, el anterior presidente de la FENOCIN, Pedro de la Cruz, ocupa cargos tanto en el gobierno como en Alianza PAÍS y en el PS-FA (Wolff, 2010: 22). En la competencia por el voto indígena, Alianza PAÍS apoya decididamente las organizaciones más pequeñas, sobre todo FENOCIN y FEINE, hecho que les ha dado mayor acceso a posiciones de poder (León, 2010: 17). León llega a una fórmula más 
drástica: "la adhesión completa de la FENOCIN al gobierno de Correa le ha proporcionado actualmente mayor espacio de acción pública y reconocimiento" (León, 2010: 16).

\section{Principios y estrategias}

Aunque la orientación sindical y de clase en la FENOCIN es constante desde su fundación, ha habido una serie de cambios profundos en sus principios. Mientras el auge de corrientes socialistas a mediados de los 1970 significó más una radicalización que un cambio ideológico (Botero, 1998: 8), las reformas de los 1990 significaron una apertura hacia el discurso étnico marcado por la CONAIE (Wolff, 2008: 116). Esto también se debe a la pérdida de importancia de los viejos grupos de interés: los huasipungueros y los trabajadores de haciendas (FENOCIN, 2004: 7). La FENOCIN se abrió hacia todos los grupos presentes en el campo, combinando su lucha por derechos laborales y tierras con demandas más abstractas, manifestadas en el concepto de la interculturalidad. Éste es entendido en términos de diversidad y fortalecimiento de las identidades étnicas (FENOCIN, 2004: 54). Se dirige contra la exclusión y el racismo implicando "cambios personales y de actitud, también cambios estructurales de la sociedad en su conjunto." (FENOCIN, s.f.: 11) En este contexto, la FENOCIN ve una lucha de dos proyectos de país: uno "del poder, de la exclusión, la rigidez y el sometimiento a los acreedores internacionales, que destruye el capital social, los valores éticos, la identidad y la confianza entre los ecuatorian@s. [...] El otro proyecto de país es el de los ecuatorian@s del pueblo, del movimiento, la participación, la inclusión y la esperanza" (FENOCIN, 2004: 27-29). La FENOCIN ve su concepto de interculturalidad como contrapuesto al concepto de plurinacionalidad de la CONAIE. Aunque sobre todo la CONAIE intenta integrar las dos propuestas, desde el principio había tensiones entre ellas que se hicieron manifiestas en la Asamblea Constituyente de 2008.

\section{EI Consejo Ecuatoriano de Pueblos y Organizaciones Indígenas Evangélicos (FEINE)}

La FEINE fue creada en 1980, en un proceso similar al de ECUARUNARI (Rodríguez, 2008: 97), como una organización de iglesias evangélicas (Wolff, 2008: 116). Los grupos religiosos aprovecharon su acceso a fondos internacionales (Tamayo, 1996: 67) y se apoyaron en las estructuras de las Iglesias Evangélicas (Barrera, s.f.: 151) para construir una organización confesional desde abajo. Guamán reparte el desarrollo de la FEINE en varias etapas que muestran su largo aliento -especialmente dada la escasa influencia de las organizaciones evangélicas hasta fines de los 1990: "configuración de organizaciones de base (1967-1979), estructuración de la organización nacional (1980-1988), irrupción de indígenas evangélicos (1989-1994), ampliación de relaciones (1995-1998), e incursión en la política partidista (1999-2002)" (Guamán, 2003: 81).

En el 1996 ya tenía 14 asociaciones provinciales (Tamayo, 1996: 67), en el 1998 crea su propio partido político Amauta Jatari, luego Amauta Yuyay, en oposición al Movimiento de Unidad Plurinacional Pachakutik-Nuevo País (MUPP-NP) de la CONAIE. A fines de los años 1990 agrupa aproximadamente a 600 iglesias locales y al 17\% de la población indígena (Wolff, 2008: 116). La FEINE, principalmente rural, se extiende a Quito y Guayaquil, especialmente por la migración indígena (Tamayo, 1996: 67). Se concentra en la Sierra central (Barrera, s.f.: 151), sobre todo en Imbabura y Chimborazo, donde sus filiales agrupan varias comunidades en las ciudades Otavalo y Riobamba (GTZ, 2004: 35-36). Ya, a mediados de los años 2000, la FEINE cuenta con 17 organizaciones provinciales y el apoyo de más de 600 iglesias locales en Chimborazo con más de 300 pastores (Andrade, 2005: 54). Según datos del CODENPE de 2007, la FEINE tiene cinco OSGs en cuatro Provincias (Chimborazo, Imbabura, Pastaza y Cañar) y un total de 26.384 miembros (IEE, s.f.). Las raíces profundas en las comunidades y estructuras evangélicas dan a la FEINE una gran fuerza organizativa que no necesariamente se ve plasmada en su posición dentro del movimiento indígena. Allí queda al margen y es vista como de afuera 
y poco auténtica, por lo cual la identificación de los indígenas con la FEINE es débil (Lucero, 2006: 39-40).

Sobre todo su incursión en la política partidaria es llamativa. Aunque Amauta Yuyay ha tenido éxito casi exclusivamente a nivel local y provincial en la Sierra ecuatoriana (Andrade, 2005: 53-54), por su participación electoral siempre ha sido acusado de dividir al movimiento indígena. Un ejemplo es el caso del anterior presidente de la CONAIE, Antonio Vargas, quien se afilió a la FEINE y a Amauta Yuyay cuando su propia organización decidió no apoyar su candidatura a la presidencia en 2002 (Freston, 2004: 144). Otra acusación es que la FEINE y su partido siempre buscan colaborar con el gobierno de turno, lo cual los pone en oposición con las demás organizaciones indígenas (Barrera, s.f.: 151). Esto se reforzó cuando Gutiérrez apoyó a la FEINE después de la ruptura con el MUPP-NP y la CONAIE para debilitarlos (Kaltmeier, 2009: 355).

La FEINE entiende su trasfondo religioso de manera política (Freston, 2004: 143) y defiende la cultura y los derechos indígenas sobre todo con proyectos de salud y de educación (Tamayo, 1996: 67). Lucero (2006: 33-34) la entiende como "evangelicalismo protestante indianizado". Sus demandas "van desde una reforma de la administración pública hasta medidas económicas que faciliten el mejoramiento de la calidad de vida de los pueblos indígenas" (Rodríguez, 2008: 97). En los últimos años, la hegemonía discursiva de la CONAIE ha dejado su huella en la FEINE, principalmente lo identitario ha sido adoptado en parte por la FEINE (Ibíd.: 41/52), como lo demuestra su cambio de nombre por el de Consejo Ecuatoriano de Pueblos y Organizaciones Indígenas Evangélicos (Ibíd.: 41).

La relación de la FEINE con los gobiernos nacionales a menudo es afirmativa (Andrade, 2005: 60). Especialmente en los tempranos 1990, los líderes solían destacar que su organización es más civilizada y fiel a las leyes que otras organizaciones indígenas, sobre todo la CONAIE (Lucero, 2006: 36). Aunque la FEINE critica al gobierno de Correa, no se une a la oposición clara que la CONAIE mantiene (Wolff, 2010: 22).

\section{La Confederación de Nacionalidades Indígenas del Ecuador}

\section{Federación de Centros Shuar}

No es sino hasta mediados del siglo XX que las iglesias empiezan una evangelización completa en la región amazónica. Para facilitar la misión, los salesianos organizaron a los Shuar en un sistema parecido al de las comunas de la Sierra, los Centros Shuar (CONAIE, 1989: 91). Estos consisten de un grupo de familias Shuar que vive en el mismo lugar. Pronto desarrollaron instituciones políticas propias integrando los religiosos salesianos como asesores, por ejemplo en la elaboración y aplicación de estatutos (Federación Shuar, 1976: 115). Poco a poco, desplazaron los religiosos del poder de sus organizaciones, adquiriendo una mayor autonomía (Barre, 1983: 179).

En los años 1960, los Centros Shuar empezaron a formar organizaciones de segundo grado en la provincia de Morona Santiago, empezando con la Asociación de Centros Shuar de Sucúa que fue ratificada por el gobierno en 1962. En 1964 formaron en un tercer nivel la Federación de Centros Shuar (Federación Shuar, 1976: 115). Esta, más tarde, participará en la fundación de la Federación de Organizaciones Indígenas del Napo (FOIN), constituida legalmente en 1969 y bajo este nombre a partir de 1973. En un principio fue miembro de la FENOC y en 1980 participó en la fundación de la Confederación de Nacionalidades Indígenas de la Amazonía Ecuatoriana (CONFENIAE) (CONAIE, 1989: 50).

Los Centros Shuar tienen como fin la defensa de su territorio y de su identidad étnica ante la colonización forzada por parte del gobierno (CONAIE, 1989: 91). Con la creciente autonomía organizativa, el entendimiento de la actividad misionera de los salesianos contribuyó a que el enfoque en lo étnico se haga más fuerte (Chiriboga, 1986: 83). Como en la región amazónica la integración en el sistema de producción agraria es baja, los Centros Shuar no se entienden 
en primer lugar en términos de clase, sino en términos étnicos y comunales (Stavenhagen, 1997: 18), lo cual contribuyó a la creación de estructuras y demandas diferentes que las de los indígenas de la sierra. De las demandas étnicas, la Federación Shuar deriva su posición defensiva contra la integración y asimilación forzada en la cultura nacional de su pueblo y por "la autodeterminación del grupo Shuar en un nuevo concepto de Estado ecuatoriano pluralista" (Federación Shuar, 1976: 129). Desde sus inicios, la Federación Shuar luchó por un Estado que reconozca la diversidad étnica y se abra hacia la diferencia. Es allí donde se encuentra por primera vez demandas para la reforma del Estado tradicional en un Estado pluriétnico y multinacional (Chiriboga, 1986: 85).

Otro enfoque importante de la Federación Shuar desde el principio ha sido su trabajo educativo. Establecieron un sistema educativo alternativo para mejorar la educación formal y para procurar una concientización de los Shuar (CONAIE, 1989: 92). En 1972 empiezan a funcionar las Escuelas Radiofónicas bilingües de los Centros Shuar (Federación Shuar, 1976: 127) que ofrecen una educación básica a distancia en las regiones aisladas.

El crecimiento de la Federación Shuar fue rápido. A mediados de los 1980 ya agrupaba a alrededor de 35.000 de los 40.000 Shuar en total, y a principios de los 1990, 263 Centros Shuar (León, 1991: 389). Participó en la conformación de la CONFENIAE (Confederación de Nacionalidades Indígenas de la Amazonía Ecuatoriana) en 1980 (Chiriboga, 1986: 84) que agrupó a nueve organizaciones regionales (Tamayo, 1996: 65) en representación de los diferentes pueblos de la Amazonía, entre ellos la Federación Shuar, la FOIN, la Federación de Nacionalidades Indígenas de Sucumbíos (FOISE), creada en 1978, y la Organización de Pueblos Indígenas de Pastaza (OPIP) (Albó, 2009: 210). La CONFENIAE tuvo -en conjunto con ECUARUNARI en la Sierra- un rol importante en la constitución de una federación indígena nacional. Aún dentro de la CONAIE, la CONFENIAE sigue autónoma y lucha muchas veces por una agenda étnica, dejando al lado las tendencias de clase. A nivel internacional ha sido miembro fundador de la Coordinadora de las Organizaciones Indígenas de la Cuenca Amazónica (COICA) en 1984 (Albó, 2009: 323).

\section{Ecuador Runacunapak Rikcharimui (ECUARUNARI)}

A partir del Concilio Vaticano II y la Conferencia Episcopal Latinoamericana de Medellín en 1968 -auge de la teología de liberación- empezó una izquierda católica a organizar y movilizar a los pobres. Pensado como un intento de frenar al comunismo, devino una involucración más profunda (ECUARUNARI, s.f.: 1). Un fruto de estos intentos era la constitución de Ecuador Runacunapak Riccharimui (El despertar de los indígenas de Ecuador) - ECUARUNARI como organización de los Kichwas ecuatorianos. Su base era el catolicismo progresivo de la diócesis de Riobamba y de movimientos laicos como el MIJAC (Movimiento Internacional de Juventudes Agrarias Católicas) (Botero, 1998: 10), a la que se juntaron algunas organizaciones socialistas. A estas corrientes se sumaron a nivel local los intentos de auto-organización de las comunidades indígenas (Simbaña 2009: 159). En junio de 1972, en la comuna Tepeyac de la provincia de Chimborazo, se realizó el congreso fundador de ECUARUNARI, con la asistencia de más de 200 delegados representantes de organizaciones indígenas de las provincias de Imbabura, Pichincha, Cotopaxi, Bolívar, Chimborazo y Cañar (CONAIE, 1989: 215). La organización se definió en este congreso como un movimiento clerical exclusivamente indígena (ECUARUNARI, s.f.: 2). Ya desde su fundación, la creación de filiales, los Huahua Riccharimui (El despertar de los hijos), fue una estrategia importante (Albó, 2009: 208). El carácter clerical se evidenció en la presencia de sacerdotes como asesores en las organizaciones provinciales. En el principio, su lucha era para la realización de otra reforma agraria con una verdadera repartición de la tierra (Simbaña, 2009: 158) y para la reivindicación de la identidad étnica de los indígenas (Tamayo, 1996: 65). Así que desde el comienzo, ECUARUNARI tenía tanto demandas de clase como demandas étnicas (Tamayo, 1996: 91). 
Los años 1970 eran marcados por la lucha por una reforma agraria completa, lo cual contribuyó a una política de alianzas con otras organizaciones con los mismos fines sobre todo con la FENOC que compartía la demanda de una repartición de tierras de ECUARUNARI (Barre, 1983: 150). Aunque ambas organizaciones tenían una ideología de clase, ECUARUANRI se diferenció de la FENOC y la ya débil FEI por sus claras demandas étnicas, llevando a un gradual cambio de un movimiento campesino de clase a un movimiento étnico indígena (Guamán, 2003: 83). A diferencia de las organizaciones sindicales, ECUARUNARI no se limitó a "una lucha contra la discriminación étnica y por la defensa de la cultura y de la lengua" (Botero, 1998: 10) -para la igualdad, en otras palabras-, sino que luchó para la ciudadanía completa de los indígenas como tales y el reconocimiento de la diferencia (ECUARUNARI/CONAIE, 1989: 40). Esto se ve expresado en un discurso de autonomía organizativa. Consideraron a las otras organizaciones indígenas, sobre todo FENOC y FEI, como manipuladas desde afuera. En esta perspectiva, sólo ECUARUNARI era una organización puramente indígena y sin influencia de movimientos o partidos no-indígenas (Moreno y Figueroa, 1992: 46-47).

La irrupción de lo étnico en la ideología de los movimientos indígenas estaba acompañada por fuertes conflictos. Sobre todo a fines de los 1970 y principios de los 1980 hubo confrontaciones dentro de ECUARUNARI. "A partir de 1977 una izquierda cristiana marxizante" (Santana, 1983: 166), con bases sobre todo en las provincias de Pichincha y Azuay de la sierra central y sur, empezó a desplazar las corrientes étnicas de la organización. A partir de 1979, ECUARUNARI estaba completamente controlada por las corrientes de clase lo cual llevó a una pérdida de sus bases, a excepción de Pichincha (ECUARUNARI, s.f.: 4). Sin embargo, la tendencia étnica no desapareció y volvió a ganar fuerza años más tarde.

Este cambio político llevó a ECUARUNARI hacia una mayor cooperación con organizaciones populares y sindicales (ECUARUNARI/CONAIE 1989: 41). En 1978 participó en la fundación del Frente Único de Lucha Campesina (FULC), después Frente Único de Lucha Campesina e Indígena (FULCI), en conjunto con FENOC y FEI y sus respectivos sindicatos (CONAIE 1989: 220/222), hecho ratificado en el Quinto Congreso Nacional de la ECUARUNARI, en Septiembre de 1979. De esta cooperación resultaron una serie de movilizaciones con demandas sindicalistas a principios de los 1980. Fue este congreso que impulsó la búsqueda de cooperaciones tanto a nivel nacional como internacional (ECUARUNARI, s.f.: 5-6). Un año más tarde fundó, junto con la CONFENIAE, el Consejo de Coordinación de Nacionalidades Indígenas del Ecuador (CONACNIE), del cual, en 1986, nace la CONAIE.

Dentro de la CONAIE, ECUARUNARI sigue muy activo. En julio de 2006 crea en Cusco, junto con la Confederación Nacional de Comunidades Afectadas por la Minería (CONACAMI) de Perú y el Consejo Nacional de Ayllus y Markas del Qullasuyu (CONAMAQ) de Bolivia, la Coordinadora Andina de Organizaciones Indígenas (CAOI). Después, incorporó organizaciones de Colombia, Chile y Argentina y fue integrada en el Consejo Consultivo de Pueblos Indígenas de la Comunidad Andina (CAN) (Albó, 2009: 324).

\section{CONAIE}

\section{El camino a la CONAIE}

La estructura de las organizaciones indígenas en los 1970 es claramente descentralizada. Existen hegemonías regionales y cooperaciones puntuales, pero ninguna organización puede asumir la representación de los indígenas a nivel nacional (Santillana y Herrara, 2009: 332). La creciente colaboración entre ECUARUNARI y CONFENIAE llevó a planes de fundar una coordinadora nacional de las organizaciones indígenas. Finalmente, se realizó en Sucúa, entre el 20 y 25 de Octubre de 1980, el Primer Encuentro de Nacionalidades Indígenas del Ecuador. Allí se decidió la creación del Consejo Nacional de Coordinación de las Nacionalidades Indígenas del Ecuador (CONACNIE) (Albó, 2009: 210), para preparar la constitución de una organización 
indígena nacional. Uno de los primeros desafíos de la CONACNIE era el de superar las diferencias existentes en las organizaciones miembros, sobre todo el conflicto entre la línea de clase de ECUARUNARI y la línea étnica de la CONFENIAE. La nueva organización nacional quiso "unir la doble dimensión de nuestra lucha, la de clase y étnica" (CONAIE, 1989: 261). Esta unión -y este conflicto- marca el desarrollo tanto de la CONACNIE como de la CONAIE hasta el presente (Barrera, s.f.: 153). A partir de 1981, ECUARUNARI -en este entonces marcadamente clasista- asumió la coordinación ejecutiva del CONACNIE (ECUARUNARI, s.f.: 6). Un objetivo de la CONACNIE era el de crear una organización regional en la Costa, donde otras organizaciones indígenas -con excepción de la FENOC- no tuvieran influencia (CONAIE, 1989: 261). La CONACNIE siguió con la política de buscar alianzas con otras organizaciones populares, como la FENOC o el FUT (CONAIE, 1989: 263).

El segundo encuentro de la CONACNIE tuvo lugar en Quito, en abril de 1984. Se declaró la independencia de todos los partidos políticos (Albó, 2009: 210). El tercer congreso del 1316 de noviembre de 1986 fue el congreso fundador de la Confederación de Nacionalidades Indígenas del Ecuador (CONAIE) (CONAIE, 1989: 33). Se definió como objetivos de la nueva organización nacional: - la devolución total de las tierras indígenas en una forma de propiedad comunitaria, - la defensa de las culturas indígenas, incluida la medicina tradicional, - la educación y concientización de los indígenas en programas de educación bilingüe en todas las lenguas indígenas del país, - la búsqueda de alianzas y financiamiento con el fin de formar un banco indígena, - la coordinación de las políticas de todas las organizaciones indígenas del Ecuador (Moreno/Figueroa, 1992: 43-44). Esta combinación de aspectos de clase y étnicos es resultado de las luchas tanto de ECUARUNARI como de CONFENIAE. Su consolidación significa un fortalecimiento más de la coalición entre las organizaciones de la Sierra y de la Amazonía que una apertura hacia otras organizaciones populares (Becker, 2008: 168), algo muy presente en la política sobre todo de ECUARUNARI de esta época. Tres años después, en agosto de 1989, la CONAIE es reconocida jurídicamente (CONAIE, 2005).

\section{Organización y desarrollo de la CONAIE}

La CONAIE está compuesta por tres filiales que representan a las tres regiones del Ecuador. Ya presentamos las organizaciones fundadoras, ECUARUNARI en la sierra y CONFENIAE en la Amazonía. Para la costa, la CONAIE impulsó la fundación de la Confederación de Nacionalidades Indígenas de la Costa Ecuatoriana (CONAICE) en 1999 (CONAIE s.f.: 6).

La primera acción en gran escala de la CONAIE ha sido el levantamiento de la fiesta del Inti Raymi en junio de 1990, considerado el mayor levantamiento indígena de la historia ecuatoriana. Los indígenas paralizaron la capital Quito por varios días y ocuparon carreteras y terrenos, además de realizar un sinfín de manifestaciones. Sus demandas estaban concentradas en un manifiesto de 16 puntos, con demandas étnicas, ciudadanas y de clase. Además de la igualdad de acceso a servicios y un apoyo especial para el campesinado, exigían el reconocimiento del carácter plurinacional del país (León, 1994: 61). Este fue el punto de partida para las otras acciones de los años 1990, que demandaron plurinacionalidad, derechos colectivos y autonomía territorial (Minkner-Brünjer, 2009: 157). En el levantamiento del Inti Raymi se utilizaron por primera vez las formas de acción que marcan las acciones de la CONAIE y del movimiento indígena hasta hoy, caracterizadas por una movilización desde las estructuras locales en el campo que se unen en jornadas de lucha y levantamientos. Así, combina los métodos de los principios del movimiento indígena, como la ocupación de territorios, con los aspectos comunitarios propios de la CONAIE y sus filiales (Minkner-Brünjer, 2009: 158). Estas formas de acción también marcaron las movilizaciones de 1992 por el cumplido de los acuerdos frutos del levantamiento de 1990, de 1994 contra las leyes de modernización agraria que querían poner un fin definitivo a la reforma agraria y la campaña por el "no" en el referéndum sobre reformas económicas y privatizaciones impulsado por el presidente Durán Ballén en 1995 (Santillana y Herrera, 2009: 335). 
Pero la CONAIE no se limitó a levantamientos y manifestaciones. Hasta mediados de los 1990 resuelve la mayoría de los conflictos de tierra que desde los 1960 eran vigentes, muchos de ellos mediante instrumentos jurídicos o políticos de bajo nivel (Simbaña, 2007: 21). Así, la CONAIE representa a los indígenas no sólo como tales, sino también en su condición de campesinos; hay elementos de clase al lado de los elementos étnicos en la política de la CONAIE.

Las movilizaciones hasta entonces, especialmente la lucha anti-neoliberal contra la política de privatización del gobierno de Durán Ballén (1992-1996), llevó a la CONAIE a una serie de alianzas con otros sectores de la sociedad civil. Esto contribuyó a la fundación de la Coordinadora de Movimiento Sociales (CMS) y -con ésta y otras organizaciones- en 1996 a la fundación del MUPP-NP - y por ende a la participación de la CONAIE en la política partidaria (Santillana y Herrera, 2009: 335). El MUPP-NP aglutinó las fuerzas indígenas de la Sierra y Amazonía y sus aliados urbanos de la CMS y una amplia gama de organizaciones de la izquierda, como los trabajadores petroleros o agrupaciones como el Foro Urbano de Quito y Ciudadanos para un Nuevo País de Cuenca (Wolff, 2010: 19). La estrategia política del MUPP-NP ha sido la "construcción de poder desde abajo" (Santillana y Herrera, 2009: 340), una orientación hacia una representación amplia del partido en el país y la participación sobre todo en los gobiernos locales. Además, mezclaron demandas étnicas como derechos colectivos y la plurinacionalidad con demandas redistributivas y anti-neoliberales (Le Quang, 2010: 112-113), razón por la cual el MUPP-NP tuvo un gran crecimiento hasta 2003.

Los conflictos entre las tendencias de clase y étnicas fueron otro factor que marcó el desarrollo de la CONAIE y del MUPP-NP. Los clasistas advocaron por una apertura hacia movimientos populares y partidos de la izquierda para posibilitar acciones mayores con el fin de mejorar la situación económica de los indígenas. La línea étnica intentaba distanciarse de organizaciones no-indígenas con el fin de crear mayores grados de autonomía en las comunidades indígenas (Minkner-Brünjer, 2009: 140). A partir de 1996 se impuso la línea étnica en la CONAIE con la elección de Antonio Vargas como presidente (Simbaña, 2008: 237). Las posiciones de clase fueron marginadas y se impuso un entendimiento de las diferencias de clase como producto de desigualdades culturales y étnicas. Es más, lo cultural fue entendido como étnico, cambiando la interpretación de una exclusión cultural y -en consecuencia- de la demanda de interculturalidad (Simbaña, 2007: 22).

A pesar de este cambio político, la CONAIE participó en la segunda mitad de la década de los 1990 en una serie de movilizaciones de índole anticapitalista o anti-neoliberal. Contribuyó en la destitución de Abdalá Bucaram 1997, influyó en la Constituyente de 1997/1998 (Santillana, 2006: 240-241), y realizó varias acciones contra el gobierno de Mahuad hasta destituirlo en alianza con sectores de las fuerzas armadas el 21 de enero de 2000. Sobre todo las acciones entre 1996 y 2002 tuvieron un trasfondo anti-neoliberal, lo que contribuyó a que la CONAIE sea percibida más claramente tanto en el ámbito nacional como internacional.

En las elecciones de 2002, la CONAIE y el MUPP-NP apoyaron a Lucio Gutiérrez, uno de los protagonistas del 21 de enero de 2000, y participaron en el 2003 en su gobierno. Como éste no respetó las demandas indígenas y siguió una administración percibida como neoliberal, en pocos meses se rompió la coalición. Este hecho es considerado el comienzo de una fuerte crisis de la CONAIE y el MUPP-NP. A partir de este momento empezaron diferentes procesos de reorganización dentro de la CONAIE. El MUPP-NP se ha "re-indigenizado", es decir, la influencia de los grupos mestizos y urbanos ha sido reducido y el partido ha sido reestructurado como brazo político de la CONAIE para recuperar el apoyo del electorado indígena (Wolff, 2010: 19). Muchos de los grupos urbanos y mestizos salieron del partido y buscaron otras afiliaciones políticas, algunos de ellos en Alianza PAÍ́S (Wolff, 2008: 126).

En los primeros años del nuevo milenio, un tema central en las acciones anti-neoliberales fue la lucha contra los planes de un Tratado de Libre Comercio con los Estados Unidos. En este contexto, la CONAIE se re-politizó y revitalizó en un sentido más clasista y en un ambiente de izquierda (Simbaña, 2009: 166). Este cambio se ve expresado en la presidencia de Humberto 
Cholango en ECUARUNARI (2003) y en la segunda presidencia de Luis Macas en la CONAIE (2004). En enero de 2005, realizaron la primera movilización desde el 2001, con unas 2.000 personas que protestaron contra el TLC (Albó, 2009).

En 2007, el CODENPE registró 804.801 personas afiliadas a la CONAIE en 141 OSG en 16 Provincias (Azuay, Bolívar, Cañar, Chimborazo, Cotopaxi, Esmeraldas, Imbabura, Loja Morona Santiago, Napo, Orellana, Pastaza, Pichincha, Sucumbíos, Tungurahua, Zamora Chinchipe) (IEE s.f.). La CONAIE se entiende como gobierno de los pueblos y nacionalidades indígenas del Ecuador (CONAIE, s.f.: 6); no sólo representa los intereses de todos indígenas, sino que niega la legitimidad de otras organizaciones indígenas que a menudo son descritas como intentos de dividir el movimiento indígena. En esta línea también caen los conflictos que había alrededor de los conceptos de interculturalidad y plurinacionalidad entre CONAIE y FENOCIN en la constituyente de 2007-2008.

Los últimos años se han visto marcados por los conflictos entre la CONAIE y el gobierno de Rafael Correa que pone en duda su representatividad. Así, las agencias estatales bajo el control parcial de la CONAIE, como CODENPE o DINEIB (Dirección Nacional de Educación Intercultural Bilingüe del Ecuador) se hicieron más débiles, mientras se crearon agencias dirigidas exclusivamente hacia las organizaciones locales o regionales, como la Secretaría de Pueblos, Movimientos y Participación Ciudadana (Wolff, 2010: 21). Estas tensiones se agudizaron en 2009 y principios de 2010 con la rotura del diálogo con el gobierno nacional por parte de la CONAIE (CONAIE, 2010: 1). Consideran a Correa un traidor y describen su proyecto político como "colonialismo del siglo XXI" (CONAIE, 2010: 2).

\section{Demandas y discurso de la CONAIE}

La CONAIE y sus filiales regionales se destacan por sus "reivindicaciones típicamente indígenas" (Le Quang, 2010: 112), que ponen énfasis en la identidad étnica dentro de un discurso que mezcla las luchas étnicas con las de clase. De hecho no se puede sostener el entendimiento que ciertas organizaciones dentro de la CONAIE sean puramente étnicas o clasistas, sino que se trata de corrientes presentes dentro de todas las organizaciones (Simbaña, 2008: 237). La mezcla de estas corrientes llevó a un conjunto de demandas que giran alrededor de los conceptos de territorio, nacionalidad, plurinacionalidad e interculturalidad.

Los nombres tanto de la CONACNIE como de la CONAIE ya delatan el entendimiento de los pueblos indígenas como nacionalidades, un desarrollo que empezó en los años 1970 y tuvo su primera expresión en el nombre de la Confederación de Nacionalidades Indígenas de la Amazonía Ecuatoriana (CONFENIAE) (Ospina, 2008: 71). Las raíces del concepto nacionalidad se encuentran en el comunismo soviético (Becker, 2008: 173). Destaca el trabajo del antropólogo ruso Yuri Zubritski que en los años 1970 procuró becas en Rusia a sus alumnos ecuatorianos, entre los cuales se encontraron Ileana Almeida, Nina Pacari y Auki Tituaña (Albó, 2009: 211). Sobre todo Almeida popularizó la teoría marxista-leninista de desarrollo que ubica la nacionalidad como etapa entre un sistema de tribus y la constitución de una nación que finalmente se transforma en estado (Ibíd.). Según la CONAIE, las nacionalidades son definidas por "un mismo origen, una historia común, idiomas propios [y] nuestras propias leyes, costumbres y creencias y formas de organización social, económica y política en nuestros territorios" (CONAIE, 2001a), además de ser más antiguas que el Estado ecuatoriano. Nacionalidad es la autodefinición de un grupo étnico (CONAIE, 2004: 116-117). Dentro de las nacionalidades hay pueblos que comparten lo anteriormente definido pero se diferencian entre sí "porque comparten una historia común, un sentido de pertenencia local y una propia forma de vivir su cultura [...]. Ocupan un territorio definido, hablan una lengua común, comparten una cultura, una historia y aspiraciones comunes" (CONAIE, 2001a). Esta definición ya es usada por la CONAIE desde los años 1980 (CONAIE, 1989: 279). En la propuesta para la Ley de Nacionalidades en 1988, la CONAIE -en conjunto con la FENOCIN y el PSE- presentó por primera vez su definición de nacionalidad 
y las reivindicaciones que deriva de ella (Ojeda, 2004: 23). El concepto de nacionalidad ha demostrado tener una gran capacidad movilizadora entre los indígenas (Becker, 2008: 173).

La nacionalidad está en estrecha relación con el concepto de territorio. Impulsado sobre todo por las organizaciones amazónicas, el territorio sobrepasa el mero significado de tierra con la inclusión de la historia y las instituciones ancestrales (ECUARUNARI/CONAIE, 1989: 39). Así, el territorio también se refiere a conceptos como la autodeterminación y la cultura del pueblo que vive allá (Ibíd., 1989: 42). El territorio es la base de las otras demandas en el contexto de la plurinacionalidad e interculturalidad (Moreno y Figueroa, 1992: 23-24). Aunque la lucha por el territorio -en lugar de la tierra- es una innovación de los años 1970, cobró especial importancia entre los años 2000 y 2005 como punto de partida de los conflictos culturales, lo cual contribuyó a una renovación de la CONAIE (Simbaña, 2008: 239). Ésta aspira a una administración autónoma de los territorios bajo el control de los pueblos y nacionalidades y la inclusión de los demás sectores de la población (CONAIE, 2001a).

Ya en la propuesta para la Ley de Nacionalidades de 1988 (Becker, 2008: 172), la CONAIE deriva de estos conceptos su demanda de una reforma del Estado (CONAIE, 1999: 51), expresada en una revisión del primer artículo de la constitución que deba incluir las nacionalidades indígenas y el carácter plurinacional del Estado (Becker, 2008: 167). Esta demanda es considerada como el "máximo objetivo" (CONAIE, 2001: 8) de la CONAIE y se mueve dentro de la demanda por una "Nueva Sociedad Intercultural" (Ibíd.), integrada por todos los sectores de la sociedad. El Estado plurinacional significa una "ampliación institucional" (CONAIE, 1999: 51-52) bajo el lema de "unidad en la diversidad" (CONAIE, 2007: 10). Se dirige contra racismo, regionalismo y colonialismo (Ibíd.: 5-10) y garantiza "el pleno ejercicio de los derechos de todas los pueblos y nacionalidades indígenas que existen dentro de un Estado" (CONAIE, 1999: 72). La plurinacionalidad es entendida como una recuperación y descolonialización del Estado, abriéndolo hacia una participación amplia de toda la población y garantizando los derechos colectivos como los individuales (CONAIE, 2007: 9). La CONAIE contrapone el Estado Plurinacional a lo que llaman "Estado uninacional" (CONAIE, 2001a), definido por la exclusión y el racismo.

Así, el Estado plurinacional es definido como "anticolonialista, antiimperialista y antisegregacionista" (CONAIE, 2001:4-5), poniendo a la "Democracia Plurinacional Comunitaria y Participativa" (Ibíd.) en el lugar de la democracia representativa mediante una transformación profunda de las estructuras del Estado. Al lado de una economía diferente propone un cambio de las estructuras jurídicas (Santillana, 2006: 241). La plurinacionalidad se refiere a un sistema de autogobiernos territoriales, una extensión de la democracia, sobre todo donde concierne el control de recursos, la pluralidad jurídica y la oficialización de las lenguas indígenas (CONAIE, 2007a: 5).

La interculturalidad es una parte importante de esta propuesta (Ibíd.: 7-8). Una sociedad intercultural es, por un lado, la condición de un Estado plurinacional, y por el otro, un tal Estado tiene que construirla mediante su apertura hacia los excluidos (Ibíd.). Es caracterizada por su estructura descentralizada y participativa (CONAIE, 2005) y la inclusión de la diferencia bajo el lema de "unidad en la diversidad" (CONAIE, 1999: 72). Se dirige contra una subordinación de unas culturas bajo otras. "La interculturalidad implica un cambio total de actitud y ello se consigue con la movilización social” (CONAIE, 2007a: 21-22).

\section{Conclusión}

La historia del movimiento indígena en el Ecuador se caracteriza sólo en sus primeras décadas como una sucesión de diferentes organizaciones con diferentes intereses e ideologías. La formación de los sindicatos que formarán la FEI a partir de 1926 indica la fuerte dependencia de todas las organizaciones nacionales de sus organizaciones de base. Su importancia declina a partir de la primera reforma agraria en 1964, que a su vez marca la formación de la FENOC 
a partir de 1965. Ésta tiene su momento más fuerte en la lucha por la segunda reforma agraria en 1973, junto con ECUARUNARI, que después forma parte de la CONAIE. Dado que las organizaciones representan a diversos grupos (la FEI a los huasipungueros, la FENOCIN a los trabajadores agrícolas indígenas y ECUARUNARI a los kichwa de la sierra), existe poca competencia -pero sí una serie de cooperaciones- entre ellas.A partir de los años 1980, marcados por la marginalidad de las organizaciones indígenas a nivel nacional debido a procesos de organización (CONAIE, FEINE) y reorganización (FEI, FENOCIN), se puede hablar de una competencia abierta entre estas organizaciones. Los momentos de cooperación (por ejemplo los levantamientos del 1990 y 1994, el derrocamiento de Bucarám en 1997 y de Mahuad en 2000) pasaron en épocas de hegemonía de una organización. Esto puede explicar el auge de actividades del movimiento indígena en los 1990 -bajo la clara hegemonía de la CONAIE- y su decadencia en los 2000, debido no sólo a factores externos -la cooperación con el gobierno Gutiérrez y Correa- sino a una hegemonía compartida entre FENOCIN, FEINE y CONAIE. Así que los años 2000 no solamente se caracterizan por una debilidad general del movimiento indígena sino por la fuerza creciente de sus diferentes organizaciones, desarrollo que complica la acción conjunta, pero favorece al crecimiento local del movimiento indígena.

TABLA 1.
\begin{tabular}{|l|c|c|c|c|c|c|c|}
\hline & 1940 & 1950 & 1960 & 1970 & 1980 & 1990 & 2000 \\
\hline FEI & $\mathrm{X}$ & $\mathrm{X}$ & $\mathrm{X}$ & - & - & & \\
\hline FENOCIN & & & $\mathrm{X}$ & $\mathrm{X}$ & - & - & $\mathrm{X}$ \\
\hline FEINE & & & & & - & - & $\mathrm{X}$ \\
\hline CONAIE & & & & & - & $\mathrm{X}$ & $\mathrm{X}$ \\
\hline
\end{tabular}

Leyenda: "Espacio blanco": ninguna actividad/no existe; “-”: algunas actividades; "X”: hegemonía organizativa 


\section{Bibliografía}

Albó, Xavier. 2009. “Movimientos y poder indígena en Bolivia, Ecuador y Perú”. En: Calderón, Fernando (Ed.): Movimientos socioculturales en América Latina. Buenos Aires: Siglo XXI, 131-332.

Albornoz, Oswaldo. 1976. Las luchas indígenas en el Ecuador. Guayaquil: Claridad.

Andrade, Susan. 2005. "El despertar político de los indígenas evangélicos en Ecuador". En: Íconos, 22, 49-60.

Barre, Marie-Chantal. 1983. Ideologías indigenistas y movimientos indios. México: Siglo XXI.

Barrera, Augusto. s.f. "Ecuador: el movimiento indígena entre lo social y lo político". En: Duterme, Bernard (Ed.): Movimientos y Poderes de Izquierda en América Latina. Madrid: Popular, 149-161.

Becker, Marc. 1997. Class and Ethnicity in the Canton of Cayambe: The Roots of Ecuador's Modern Indian Movement, http://www.yachana.org/research/diss.pdf (27.7.2011).

2008. Indians and Leftists in the Making of Ecuador's Modern Indian Movements. Durham/London: Duke University.

Botero, Luis Fernando. 1998. "Estado, cuestión agraria y movilización india en Ecuador”. En: Nueva Sociedad, $153,56-72$.

Bretón, Víctor. 2003. "Desarrollo rural y etnicidad en las tierras altas de Ecuador". En: Bretón, Víctor/García, Francisco (Ed.): Estado, etnicidad y movimientos sociales en América Latina. Ecuador en crisis. Barcelona: Icaria, 217-253.

Büschges, Christian. 2009. "Políticas de identidad entre integración y autonomía: movimiento indígena, sociedad y Estado en Ecuador y Nepal desde una perspectiva comparada y transnacional”. En: Ospina, Pablo/Kaltmeier, Olaf/Büschges, Christian (eds.): Los Andes en movimiento. Quito: Corporación Editora Nacional/Universidad Andina Simón Bolívar, 41-63.

Chiriboga, Manuel. 1986. “Crisis económica y movimiento campesino e indígena”. En: CLACSO/ILDIS (eds.): Movimientos sociales en el Ecuador. Buenos Aires/Quito: CLACSO, ILDIS, 63-91.

CONAIE.1989. Las nacionalidades indigenas en el Ecuador. Nuestro Proceso organizativo. Quito: CONAIE, Abya-Yala.

1999. Las Nacionalidades Indígenas y sus derechos colectivos en la Constitución. Quito: CONAIE.

2001. "Proyecto Político de las Nacionalidades y Pueblos del Ecuador". En:<http://www.conaie.org/congresosde-la-conaie/ii-congreso-ed-la-conaie/99-proyecto-politico-de-las-nacionalidades-y-pueblos-delecuador?format $=$ pdf $>(12.9 .2010)$.

2001a. "Proyecto Político de la Confederación de Nacionalidades Indígenas del Ecuador (CONAIE)". En: < http:// www.llacta.org/organiz/coms/com862.htm> (4.10.2010).

2004. Propuesta de Ley de Biodiversidad. Quito: CONAIE.

2005. "Constitución de la Confederación de Nacionalidades Indígenas del Ecuador". En: <http://www. nacionmulticultural.unam.mx/declaraciones/docs/decl_031.pdf $>$ (25.10.2010).

2007. Propuesta de la CONAIE frente a la Asamblea Constituyente. Quito: CONAIE.

2007a. "La CONAIE frente a la Asamblea Constituyente". En: <http://www.cebem.org/cmsfiles/archivos/ principios-lineamientos-conaie.pdf $>$ (30.9.2010).

2010. "Declaración al pie de taita Imbabura y mama. Los Pueblos y Nacionalidades Indígenas del Ecuador frente a la Cumbre de los Presidentes del ALBA". En: <http://movimientos.org/imagen/CONAIE.pdf> (25.10.2010).

s.f. ¿Qué es la CONAIE? El 22 de Octubre del 2007. Quito: CONAIE.

ECUARUNARI/CONAIE. 1989. "Lineamientos políticos generales del movimiento indígena en el Ecuador". En: ECUARUNARI, FENOC, ALAI (eds.): Nuestra voz, nuestra cultura. Quito: ECUARUNARI/FENOC/ ALAI, 38-42.

ECUARUNARI. s.f. "Proceso Organizativo de ECUARUNARI”. En: <http://ecuarunari.org/portal/info/historia > (20.11.2010).

Federación de Centros Shuar. 1976. Solución original a un problema actual. Sucúa: Federación Shuar.

FEI. s.f. "Federación Ecuatoriana de Indígenas". En: <http://www.cte-ecuador.org/> (10.10.2010).

FENOCIN. 2004. Noveno Congreso Ambato, 20-22 de mayo de 2004. Quito: FENOCIN.

s.f. "Consolidación organizacional, Revolución agraria, Interculturalidad, Soberanía alimentaria, Construcción del Socialismo". En: <http://www.uasb.edu.ec/UserFiles/380/File/Presentacion\%20FENOCIN\%202009 Patricio\%20Sandoval.pdf $>$ (25.9.2010).

Freston, Paul. 2004. Protestant Political Parties. A Global Survey. Hampshire/Burlington: Ashgate. 
GTZ. 2004. Estudio sobre la Cooperación Alemana con Nacionalidades y Organizaciones Indígenas en Bolivia, Ecuador y Guatemala, Tomo 4: Anexos del Estudio de Ecuador. s.1.: GTZ.

Guamán, Julián. 2003. Indígenas evangélicos ecuatorianos. Quito: FEINE.

Instituto de Estudios Ecuatorianos (IEE). s.f. Mapeo, Actores y Poder. Anexos. http://www.iee.org.ec/ investigaciones/MAPEO\%20actores\%20y\%20poder/actorespoder_anexo.pdf (3.5.2011).

Kaltmeier, Olaf. 2009. "Das Land neu gründen. Gesellschaftliche Kontexte, politische Kulturen und indigene Bewegungen in Südamerika". En: Mittag, Jürgen/Ismar, Georg (eds.): ¿ "El pueblo unido"? Soziale Bewegungen und politischer Protest in der Geschichte Lateinamerikas. Münster: Westfälisches Dampfboot, 339-363.

León, Jorge. 1991. "Las organizaciones indígenas: Igualdad y diferencia. La afirmación de los conquistados". En: Cornejo, Diego (ed.): Indios. Una reflexión sobre el levantamiento indígena de 1990. Quito: ILDIS, El Duende, Abya-Yala, 373-417.

1994. De campesinos a ciudadanos diferentes. Quito: CEDIME/Abya-Yala.

2010. "Las organizaciones indígenas y el gobierno de Rafael Correa”. En: Íconos, 37, 13-23.

Le Quang, Matthieu. 2010. "La reestructuración de la izquierda en Ecuador. Del movimiento indígena hasta Rafael Correa”. En: Gaudichaud, Franck (Ed.): El volcán latino-americano. http://alainet.org/images/El_ volcan latino FG.pdf (5.10.2010), 111-120.

Lucero, José Antonio. 2006. "Representing 'Real Indians'. The Challenges of Indigenous Authenticity and Strategic Constructivism in Ecuador and Bolivia". En: Latin American Research Review, 41 (2), 31-56.

Minkner-Brünjer, Mechthild. 2009. "Zwischen Erfolgen und Ausbootung. Soziale Bewegungen in Ecuador". En: Mittag, Jürgen/Ismar, Georg (Eds.): ¿”El pueblo unido”? Soziale Bewegungen und politischer Protest in der Geschichte Lateinamerikas. Münster: Westfälisches Dampfboot, 133-165.

Ojeda, Lautaro. 2004. "Análisis politológico del proceso de construcción de la autonomía multicultural en el Ecuador”. En: http://www.latautonomy.org/EstudioPolitico_Ecuador.pdf (22.7.2010).

Ospina, Pablo. 2008. "La deriva de una promesa. Movimientos sociales, democracia y neoliberalismo". En: Andrade, María/Herrera, Gonzalo/Ospina, Pablo (2008): Mapa de movimientos sociales en el Ecuador. $<$ http://www.iee.org.ec/investigaciones/MOVIMIENTOS\%20SOCIALES/movimientosSOCIALES.pdf> (24.8.2010).

Rodríguez, Héctor. 2008. Gobernanza local en Ecuador, un proceso visto desde la comunidad. México: FLACSO.

Sánchez, Francisco y Freidenberg, Flavia. 1998. "El proceso de incorporación política de los sectores indígenas en el Ecuador. Pachakutik, un caso de estudio.” En: América Latina Hoy, 19, 65-79.

Santana, Roberto. 1983. Campesinado indígena y el desafío de la modernidad. Quito: CAAP.

Santillana, Alejandra. 2006. "Proceso organizativo y límites del proyecto político de Pachakutik". En: Ospina, Pablo (Ed.): En las fisuras del poder. Quito: Instituto de Estudios Ecuatorianos, 215-265.

Santillana, Alejandra y Herrera, Stalin. 2009. "Génesis, experiencia, transformación y crisis del Movimiento Indígena Ecuatoriano”. En: Coscione, Marco (Ed.): América Latina desde abajo. Quito: Abya-Yala, $330-352$.

Simbaña, Floresmilo. 2007. "El movimiento indígena y el actual proceso de transición". En: América Latina en movimiento, 423, 21-24.

2008. "Los derechos culturales y colectivos en la nueva constitución de Ecuador”. En: Peñaranda, Raúl (ed.): Democracia, Interculturalidad, Plurinacionalidad y Desafios para la Integración Andina. La Paz: CEBEM, 237-242.

2009. "El movimiento indígena ecuatoriano y la construcción de la plurinacionalidad". En: Hoetmer, Raphael (ed.): Repensar la politica desde América Latina. Lima: Facultad de Ciencias Sociales, 153-167.

Stavenhagen, Rodolfo. 1997. "Indigene Völker: Neue Akteure in Lateinamerika”. En: von Gleich, Utta (ed.): Indigene Völker in Lateinamerika: Konfliktfaktor oder Entwicklungspotential?. Frankfurt a.M.: Vervuert, 1533.

Tamayo, Eduardo. 1996. Movimientos sociales. La Riqueza de la Diversidad. Quito: ALAI.

Verdesoto, Luis. 1986. "Los movimientos sociales, la crisis y la democracia en el Ecuador". En: CLACSO/ILDIS (eds.): Movimientos sociales en el Ecuador. Buenos Aires/Quito: CLACSO, ILDIS, 13-61.

Wolff, Jonas. 2008. "Buscando respuestas a la doble transformación: El movimiento indígena ecuatoriano". En: de la Fontaine, Dana/Aparicio, Pablo (Eds.): Diversidad cultural y Desigualdad social en América Latina y el Caribe. S.L.: Heinrich Böll Stiftung, 113-129.

2010 "Elitenwandel in Ecuador". En: <http://library.fes.de/pdf-files/iez/07116.pdf> (21.9.2010). 
